\title{
Chapter 7:
}

Applying psychological theory to in-game moral behaviors through the development of a purpose-made game

\author{
Sarah E. Hodge \\ John McAlaney \\ Christos Gatzidis \\ Eike Falk Anderson \\ Davide Melacca \\ Jacqui Taylor \\ Bournemouth University
}

\begin{abstract}
A number of video games involve moral narratives or require the players to make moral decisions. Research from psychologists has helped to understand the effects that video game content can have on how individuals think, feel and behave. Recent research has examined the role of morality in video games, yet there are many inconsistencies in the findings that could be due to the use of commercial video games for research purposes, which contain biases such as familiarity with the game and favorite characters. By developing a bespoke game designed specifically for the purpose of exploring morality, these potential biases can be reduced. Before designing the game, morality in existing video games is critically analyzed, using theories from moral psychology. From this, a game was developed to measure behavioral outcomes through which moral decisions are made; with the aim to address biases that are inherent in commercial games. Then, the resultant game was used to investigate how participants make moral choices in video games.
\end{abstract}




\section{Applying psychological theory to in-game moral behaviors through the development of a purpose-made game}

Much-publicized concerns have been raised in the past about the moral content of video games, such as the Grand Theft Auto series (GTA; Rockstar, 1997-2015), which includes content and activities such as nudity, prostitution, guns, drug dealing and driving recklessly. Kocurek (2012) draws a parallel between GTA and Death Race (Exidy, 1976), an earlier game that raised considerable controversy, as they are both based on similar acts of violence and moral violations (such as running over pedestrians and damaging other vehicles). Furthermore, Kocurek (2012) explains that the reason Death Race received this controversy is due to the type of violent content it contained. Violent content which is related to war is more socially and culturally acceptable as it is a part of history, whereas violence towards innocent people, such as running over pedestrians, would be outside the social and cultural norms (McKernan, 2013).

It is important to note that Bowman (2016a) reports on how even early media and technology, including telephones and comic books, have caused controversy. However, one of the reasons for the specific controversy around video games is that, unlike other forms of media such as films, video games are more interactive and include a behavioral component. Bowman (2016a) highlights that it is the interactivity and the simulations of these behavioral acts that render video games controversial, particularly due to the violent content such as, notably, acts of murder. As video games have advanced as a medium, so have the graphical depictions and possibilities within them, creating this way additional concern over the content and the behaviors carried out in these virtual worlds. Games move beyond other media by not simply showcasing moral violations, but also providing players with the opportunities to both encounter morality and respond to it. Jesse Schell, a pivotal game designer, described how video game design needs to evolve to allow for the prospect of the medium to produce the 
equivalent of Shakespearean work, a notable example of literature (Miller, 2013). GTA has been suggested to be focusing more on moral themes within the gameplay (Oliver et al, 2015). In this spirit, and in reflection of the increased maturity of moral themes in video games (Limperos, Downs, Ivory, \& Bowman, 2013; Oliver et al, 2015) for the following part of this chapter we look to discuss the research, specifically related to the measuring of moral considerations of the players through designing and creating a bespoke video game.

\section{Agency, interactivity and behavior: Past research}

Thomas (2006) notes that, compared with other media, there is a different level of involvement, agency and interactivity in videogames. Agency is the feeling produced from the player's perception of the level of control or choice they have in a video game (Frasca, 2001). For example, this highlights the difference between watching someone killed onscreen (i.e. a character being killed) and the individual taking action by pressing a button to kill the character. Thus, many video games provide the player the opportunity to make decisions and have in-game behavioral outcomes. These behavioral outcomes are a fundamental part of the video game experience. Agency is therefore not only unique to the medium of video games, but also directly related to the understanding of in-game behaviors. Other researchers have suggested that interactivity, as per above, but also presence too, are fundamental parts of video games and are aspects of what makes video games different from other forms of media (Grodal, 2000; Tamborini \& Bowman, 2010). Interactivity has been defined as the degree to which the users (i.e. the players) can change and control the 'form' and 'content' within the environment (i.e. the game; Steuer, 1992). Other definitions have distinguished between the interaction produced by the player (i.e. interactivity as process) and the system/game (i.e. interactivity as product; Stromer-Galley, 2004). Salen \& Zimmerman (2004) highlight the many definitions of interactivity, but similarly suggest it is the relationship between the game and player, as well as the choices the players make within the 
game. This interactivity in video games contributes to the player's' experience of immersion and engagement within the virtual world. Oliver et al., (2015) provide the example of how this can take place through the role of crying in video games, an action that the player could both cause and resolve. There has been much debate around the terminology used to describe immersion within video games. Brockmyer et al., (2009) suggest the term engagement encompasses the components of immersion (Frasca, 2001), presence (Tamborini \& Bowman, 2010) and flow (Sherry, 2004). While an extended discussion of these constructs is beyond the scope of this chapter, for balance we will use engagement to broadly describe these phenomena. Bowman (2016b) in particular highlights the role of the behavioral demand; as, in order to progress in video games, the player is required to make choices through pressing buttons, which are not only represented in the game play but also through the game's set-up (such as navigating the menus). This process of making choices does also overlap with the cognitive demand, through the thinking required about the choice(s) and consequently the button(s) pressed. Bowman, Weber, Tamborini, and Sherry (2013) demonstrated this overlap between cognitive and behavioral demands of the game, through the connection between skill and performance in video games.

Gameplay effects. Much of the research into post-gameplay effects such as aggression has made the connection between in-game behaviors transferring into real life behavior (APA, 2015). This has produced a long-standing debate within the literature as to whether this exists or not. It has been suggested that when an individual is in a virtual space, real life norms may not apply and therefore this could explain virtual behavior, especially for those behaviors that would be considered morally violating in real life. This further connects to the concept of a magic circle (Huizinga, 1949). Applying this concept infers that video games are not constrained by the norms and rules of real life but, instead, are governed by the rules of the game that exist within it (Salen \& Zimmerman, 2004). Thus, the implications of 
these behaviors do not apply to real life and are therefore not of concern. However, Consalvo (2009) argues against the existence of a magic circle, due to the overlap of real life and the gaming world, as players bring in real life aspects into the game, such as expectations and interpretations from the gaming world. Meta-analytic work by Anderson et al., (2010) suggests problematic associations between violent gaming and aggression, yet work from Sherry (2001) promotes smaller effects than similar research on television violence. Finally, Ferguson (2007) suggests that publication biases might inflate any effects of violent gaming. This has also led to the investigation of violent video games, aggression and prosocial behavior, with the results suggesting a mixture of a negative relationship (Prot et al., 2014) and no relationship (Jerabeck \& Ferguson, 2013) with prosocial behavior. Regardless, there is a continued debate around behavior in video games and, accordingly, the role of moral behavior.

Gaming and morality effects. Research has started to investigate how an individual's sense of morality in real life might relate to morality whilst playing video games. Much of this research has done so through the use of commercial games, due in part to recent patterns in game development and design that have favored the inclusion of more morally engaging and less hedonic moral decision-making, as discussed previously (Christiansen, 2017: Miller, 2013).

However, there are inconsistencies in the findings from the research using commercial games. For example, Weaver and Lewis (2012) examined moral decisions made in a video game using Fallout 3 (Bethesda, 2008), and the Moral Foundations Questionnaire (MFQ; Graham, Haidt, \& Nosek, 2008) based on the Moral Foundation Theory (MFT; Haidt \& Joseph, 2004, 2007). Weaver and Lewis (2012)observed that moral decisions in a video game were similar to moral decisions made in real life, similar to Consalvo's (2009) critiques of the magic circle assumptions that players "leave behind" reality when entering a gaming space. 
In contrast, Hartmann and Vorderer (2010) examined the role of enjoyment and violent video games using the game Half-Life 2 (Valve Software, 2004). The results suggested that players were morally disengaged, that is players appeared to selectively dissociate from the behavior that violates an individual's moral codes (Bandura, Barbaranelli, Caprara, \& Pastorelli, 1996). The results also suggested that more familiarity with the game resulted in less negative affect and guilt, and greater enjoyment. The authors suggest that it is through the mechanism of moral disengagement that potential immoral behavior could be carried out without detriment to enjoyment. Essentially, it could be suggested that players engage the "magic circle" of the game space, as harboring too many expectations of reality would hinder enjoyment of the action. Grizzard, Tamborini, Sherry, and Weber (2016) used Call of Duty: Modern Warfare 3 (Activision, 2015) and Operation Flashpoint (Bohemia Interactive, 2001) and observed that repeated exposure to the in-game violence reduced feelings of guilt during game play; that is players felt less guilty when committing violent acts as they gained more experience with the game's content. The study incorporated the use of two commercial games which potentially improves the generalizability of its findings to other video games.

As shown above, there are inconsistencies regarding the role of moral behavior in video games, with some studies showing players to react with moral sensitivity and others showing players to suspend morality during gameplay. Yet, these inconsistencies could be due to methodological issues with using commercial video games as these contain biases such as familiarity with the game and favorite characters. Connected to familiarity is another potential bias, that of re-playability of the game; if and how much the game has been replayed and the amount of experience the player already has with the game itself. Some of these limitations were addressed in part by Grizzard, Tamborini, Lewis, Wang, and Prabhu (2014), who examined if being bad in a video game related to feelings of guilt and moral 
salience whilst playing a modified first-person shooter. Participants were either assigned to a memory recall task (either guilty memory or ordinary memory) or played a video game (either a guilt inducing level playing as a terrorist soldier, or a non-guilt inducing level playing as a UN soldier). After gameplay, the MFQ (Graham et al., 2008) and a three-item guilt measure were completed by participants. The results suggested participants playing as terrorists felt significantly more guilt than those who played as UN soldiers. This was significantly associated with two of the five MFT foundations of Care/Harm (propensity for caring and dislike of harm to others) and Fairness/Cheating (desire for fairness and dislike of cheating), though not with the remaining three foundations of Loyalty/Betrayal (group membership and dislike of betrayal), Authority/Subversion (respect for authority and tradition) and Sanctity/Degradation (relating to purity and contamination) (Haidt \& Joseph, 2004, 2007). Grizzard et al., (2016) suggest that antisocial behavior in video games could relate to prosocial behavior as the participants who violated the foundations could become more morally sensitive (as represented by higher ratings of guilt). Modifying existing commercial games ensures the game being played is similar to what participants would normally be experiencing and playing. However, there are still restrictions with this approach in terms of content that cannot be modified.

Joeckel, Bowman, and Dogruel (2012) developed a game to specifically examine the role of morality using the same MFT foundations, as considered in Grizzard et al., (2014). The game was made using the Aurora engine within the game Neverwinter Nights (BioWare, 2002), a toolset which gave players the opportunity to develop their own adventure game scenarios (Greig, Muzyka, Ohlen, Oster, \& Zeschuk, 2002). Such a procedure follows the recommendations of Elson and Quandt (2014), associated with using heavily customized versions of off-the-shelf video games. Six scenarios were created: five related to the moral foundations from Grizzard et al., (2014) and a sixth one, which was a foil scenario, used to 
reduce demand characteristics of players who might uncover that each scenario was specifically aimed at one particular aspect of morality (the sixth scenario, an amoral event, only involved a book falling accidentally into some mud:

http://onmediatheory.blogspot.com/2012/03/morality-in-video-games-gut-or-game.html). Each scenario asked the player to interact with a NPC (Non-Player Character, i.e. a computer-controlled entity similar to the human-player's avatar in the game) who would share their troubles (similar to many popular role-playing games), and then asked the player to help make a choice to either violate or uphold the foundation connected with the scenario. The authors observed that moral foundations in the game were upheld when the particular moral foundation was salient to the player (salience is the importance of the foundations, the most salient foundation for all players in these results being Harm/Care), whereas non-salient foundations resulted in random, amoral decisions (Joeckel, Bowman, \& Dogruel, 2013). The latter finding was compelling in that there was no evidence that players with low moral salience actively violated morality in-game. This is a finding in line with Lange (2014), who suggests that players have a preference for the 'good option' and tend to avoid moral violations for fear of in-game reprisal. Tamborini et al., (2016) in a later study found similar results for the foundations that were upheld and violated. In Joeckel et al., (2013), developing a novel video game did reduce some of the biases, but a number of limitations were still present. For example, the moral decisions presented to players (and even the scenarios themselves) were text-based, which might not have been as engaging as using spoken dialogue. Also, the player was asked if another character should violate a scenario rather than the player violating a scenario themselves, thus the lack of agency could have been an issue. Some of these limitations could be attributed to restrictions within the Aurora engine but, nonetheless, they speak to a gaming experience that might have fallen short in terms of what modern gamers expect. Likewise, the Neverwinter Nights game was already 
nearly 10 years old at the start of their study, which might have appeared somewhat dated to participants. Drawing on both the previous research and concepts discussed led us to the development of the current project which will be discussed in the next section of this chapter.

\section{Creation of a theory-driven bespoke morality game}

This work described in the remainder of this chapter aims to draw from the disciplines of psychology and game development in order to create a game to measure morality. A purpose-made or bespoke game refers to a game that is designed and created with and/or to a specific specification. Creating and designing games for research allows for some of the biases of commercial games to be addressed, such as familiarity with the game and favorite characters. Therefore, using game engines to create games or similar Interactive Virtual Environments (IVEs) is well-suited to research (cf. Elson \& Quandt, 2014), as such systems provide complete control over every aspect of the virtual world and, accordingly, the variables that determine these aspects, which in turn allows for further rigor (Lewis \& Jacobson, 2002). Thus, the aim of this purpose-made game was to create a stronger sense of moral agency and, at the same time, produce an experience similar to what participants would normally expect to have from traditional gameplay.

Notably, the function and focus of a purpose-made game is not primarily for entertainment, similar to the concept of serious games. Serious games are developed for a purpose and function other than entertainment, usually with a strong focus on learning and behavior change (Connolly, Boyle, MacArthur, Hainey, \& Boyle, 2012). Although taxonomies of serious games have been proposed (e.g. De Lope \& Medina-Medina, 2016), taxonomies tend to focus on the player outcomes (such as the assessment and feedback)

rather than the measured outcomes for researchers (De Lope \& Medina-Medina, 2016). Whilst creating purpose-made games can on occasion fit the definition of serious games creation, due to the function being other than entertainment, when the focus of the design is 
on measuring psychological phenomena, this could be suggested to be taking a different approach to the definition of serious games previously mentioned by Connolly et al., (2012) and the taxonomies of serious games (De Lope \& Medina-Medina, 2016). So, rather than developing games to invoke change in participants, the focus in this case is on developing a game to observe and record current behavior. Therefore, purpose-made games could be utilized more within research, specifically for the measurement of psychological phenomena.

When creating a purpose-made game, an important consideration is the similarity to commercial video games. If the resultant game is very different from the commercial games participants would normally be playing, then, the question of how generalizable the results really are would need to be considered. This can be addressed at the design stage as well as through comparing participants' experiences with commercial and purpose-made games. At the design stage, research into commercial games supported the development of the game through representing key features of current commercial games, such as choice mechanics. During the testing phase of the game, the participants' levels of engagement for the purposemade game and commercial games were measured. Thus, a comparison was drawn between commercial games and the purpose-made game. This can then support the understanding of the aspects and factors that influence game behavior. For example, familiarity with the game has been previously found to influence affective outcomes (Hartmann \& Vorderer, 2010). Therefore, a purpose-made game would be able to control for familiarity. This allows for the outcomes of commercial and purpose-made games to be compared. Additionally, this also has implications for the research methodology used in video game research and, specifically, for measuring behavior outcomes.

Measuring moral intuitions and decision making. A literature review was conducted in order to identify theories of morality relevant for application within a video 
game. Mentioned earlier in this chapter, MFT was chosen as it has been used previously in related research (Weaver \& Lewis, 2012; Joeckel et al., 2013; Grizzard et al., 2014) and scenarios could be developed from the moral foundations, as was specifically done in Joeckel et al. (2013) and used in later work by Tamborini et al (2016). These moral foundations include the previously mentioned five-Care/Harm, Fairness/Cheating, Loyalty/Betrayal, Authority/Subversion and Sanctity/Degradation (Haidt \& Joseph, 2004, 2007), plus the recent additional sixth foundation, that of Liberty/Oppression (relating to dominance and dislike of bullies; Haidt, 2012). These six foundations were useful for video game research, as the foundations are prevalent in many commercial video games (examples of games are provided in the next section) as well as providing structure of the moral domain, to be able to create new scenarios. Furthermore, MFT suggests the process of moral decision making happens through both; moral intuitions that are quick gut responses and by deliberative rational responses (Haidt, 2001). The MFT has been incorporated into the Model of Intuitive Morality and Exemplars (MIME; Tamborini, 2011; Tamborini, 2013), a multi-stage model of how media content is processed and evaluated by the individual. This model is useful to apply to video games, as it highlights the reciprocal relationship between the individual and the environment, with specific focus on the influence of media. The model includes the process of decision making through short-term and long-term components, and incorporates MFT and intuitive and rational decision making.

In addition to this, Hartmann (2011) suggested two decision making systems for virtual decisions: one system is experiential (automatic, fast and intuitive) and the other system is rational (reflective, slower and cognitive). Hartmann (2011) states that the rational system could be responsible for an individual's assessment of reality, i.e. if the actions could happen in real life, then the realism of the game is assessed by the individual. The experiential system is more primitive, in so far that what is experienced and perceived is 
believed. Although Hartmann (2011) provides examples of how both intuitive and rational systems could be activated, questions still remain around the decision-making process being intuitive or rational during gameplay. Therefore, this decision-making process is important as it demonstrates the behavioral and, accordingly, cognitive aspects of playing video games. Previous research has suggested that in video games people make these intuitive decisions for salient foundations, whereas non-salient foundations suggested amoral and random decisions (Joeckel et al., 2013). On this, Bowman (2016b) suggested that more research is needed to understand the role of moral decision-making in video games. Due to the previous results from the literature suggesting differences within each of the foundations and the dual theory of decision making, response times of the decisions appear to be an important consideration for the measurement of the intuitiveness of a moral decision (Tamborini et al., 2016).

\section{The development of the moral decisions in the game.}

In order to create moral content and decisions for our bespoke game, we analyzed around 30 previous commercial video games that included morality in the form of a narrative theme and/or the requirement of making moral choices. The following titles were shown to be particularly relevant: Until Dawn (Supermassive Games, 2015), the Fable series (Lionhead Studios, 2004-2014), the BioShock series (2K-Games, 2007-2013), and Spec Ops: The Line (2K Games, 2012). These titles were selected as each contained moral content and choices that could be matched to each of the MFT foundations (i.e. BioShock's 'Little Sisters' and Care/Harm foundation), plus they also demonstrated morality in video game design (i.e. Spec Ops: The Line and Until Dawn how choices were designed and made in the game). Specifically, the analysis included what choices are presented to the player in the game plus what the consequences of these choices are (if applicable). Moral psychology theories were then used to evaluate and design game content accordingly, including the aforementioned MFT (Haidt \& Joseph, 2004) and moral disengagement (Bandura et al., 1996). In addition to 
this, we considered that morality in video games is, for the most part, absolute (clear and certain choices), utilitarian (choices that involve thinking about the majority), and focused on the action (the outcome of the choice; Heron \& Belford, 2014). Players are often presented with binary decisions in these games, and thus, binary decisions were identified as a typical feature of many video games and incorporated into the design of our purpose-made game.

Developing the moral decisions for the game required the above considerations, in order to create scenarios for each of the six foundations mentioned previously. Scenarios were developed in the form of vignettes to be foundation-specific; with only one foundation being triggered so that each moral foundation could be examined individually (similar to what was done by Joeckel et al., 2013 and Tamborini et al., 2016). More than one scenario was created for each foundation to pilot extra scenarios in the case of a failed manipulation; such as more than one foundation activating.

To create vignettes, we considered prior work from Clifford, Iyengar, Cabeza, and Sinnott-Armstrong (2015), who created vignettes for each of the six MFT foundations. However, their vignettes were not appropriate to use due to having too many extraneous contextual details, such as references to family in scenarios that were not the Loyalty/Betrayal foundation (family being a critical component of Loyalty/Betrayal according to Haidt \& Joseph, 2004). Also, the vignettes contained a considerable amount of gender-referential information, whereas we did not want to bias participants in our study. However, for the Care/Harm foundation, Clifford et al (2015) focused on two types of Care/Harm scenarios, emotional (i.e. 'You see a teenage boy chuckling at an amputee he passes by while on the subway') and physical (i.e. 'You see a woman throwing a stapler at her colleague who is snoring during her talk'). Due to the prevalent nature of harm in video games, physical harm was chosen. Dill, Gentile, Richter, and Dill (2005) found that $60 \%$ of the top selling games at the time contained violence. These vignettes were then used to 
generate new scenarios for our bespoke game.

Vignettes were selected to keep the text short yet informative. "You see" was added to the beginning of scenarios for the pilot as this would help the participants to create an image of a scenario from the written text. Each scenario and decision followed the same format to reduce biases, thus, the only difference was the content of the moral foundation. The scenarios were created with considered caution for the reduction of biases from situational cues. Also, the scenarios were developed within a word count (18-20) and character count (95-110) boundary. This was the same for the options presented for the choice, which both had a word count (7-10) and character count (39-58) boundary. This was in order to control for potential differences between each of the scenarios. The situations within the scenarios were developed to be unresolved and in need of resolution by the player. The scenarios were developed in this way for three reasons; the first being that if the situation was created to be too much of a violation, then the response could be different compared to a response to a triggering situation. As an example of the difference between violating and triggering for the Loyalty/Betrayal foundation; the trigger would be group membership, whereas a violation would be something which betrayed the group. The difficulty was creating scenarios that triggered the specific moral foundation enough, without however being violating. Furthermore, scenarios that are highly violating, such as killing a child, would connect to moral taboos, which in turn could create another confound with the decisions made in the game (Young, 2013). Secondly, it meant that the player was part of the resolution and connects to their agency; this is because an on-screen behavioral outcome was required through making a choice. Third, if a violation has already taken place, then this would have compromised the choice, to violate or not, as this has already happened and, thus, removed player agency. Therefore, scenarios (listed in Table 1) were created to be as neutral as possible in order for choice to be required, and each involved at least one NPC. 
Table 1. Moral scenarios for theory-driven bespoke game

\begin{tabular}{|l|l|}
\hline \multicolumn{1}{|c|}{ Moral foundation } & \multicolumn{1}{c|}{ Scenario description } \\
\hline Care/Harm & An injured NPC \\
\hline Fairness/Cheating & Two NPCs sharing coins \\
\hline Loyalty/Betrayal & A group of NPCs with a promise between them \\
\hline Authority/Subversion & An order from an NPC General \\
\hline Sanctity/Degradation & An NPC trying to fix a leaking sewage pipe \\
\hline Liberty/Oppression & An NPC trying to fix a free speech sign \\
\hline
\end{tabular}

The development and programming of the game. The development of the game was part of a University-funded project as a form of collaboration with the relevant Departments in the institution. For this, an undergraduate research assistant was hired to program the game. The Unreal Engine version 4 (UE4) was selected as the development platform, i.e. the infrastructure hosting the IVE, as it best fit the experience of the development team (Anderson et al., 2013) and the requirements of the project. UE4 is a contemporary and popular tool for game and IVE creation and the nature of the system meant that the prototype produced would still be playable and editable in the future (whereas other less supported engines can result in potential incompatibility). Many commercial games have used the Unreal Engine since its inception in the late 1990s, with some examples including the Unreal Tournament series (Epic Games, 1998-2007), Spec Ops: The Line, the BioShock and Mass Effect series (BioWare, 2007-2012) and many others. In 2014 the Guinness World Records, named the Unreal Engine the most successful game engine which was attributed to the development of 408 games using this tool (Guinness World Records, 2014). The game development team constructed the IVE based on a real life environment; namely a University type environment. This environment was created to appear similar to the real world and reasonably photorealistic for believability in order to compare video game choices with real life moral reasoning (see Figure 1). The objective of this was to support realism of the game environment for the players and to encourage engagement and social presence within the game (Tamborini and Bowman, 2010). The design and interface of the game is similar to a 
first-person Role Playing Game (RPG). This was selected for its similarity to commercial video games that contain moral choices which were discussed previously, such as the BioShock series. The genre also was suitable for the purpose of creating scenarios within a game environment. This is due to the characteristics of these games having strong narrative structures, again, as seen in commercial games such as Until Dawn.

Figure 1. Screenshots of the purpose-made IVE for each of the scenarios from the players point of view (Top left: Liberty/Oppression, top right: Care/Harm, middle right: Authority/Subversion, bottom right: Sanctity/Degradation, bottom left: Loyalty/Betrayal, and middle left: Fairness/Cheating).

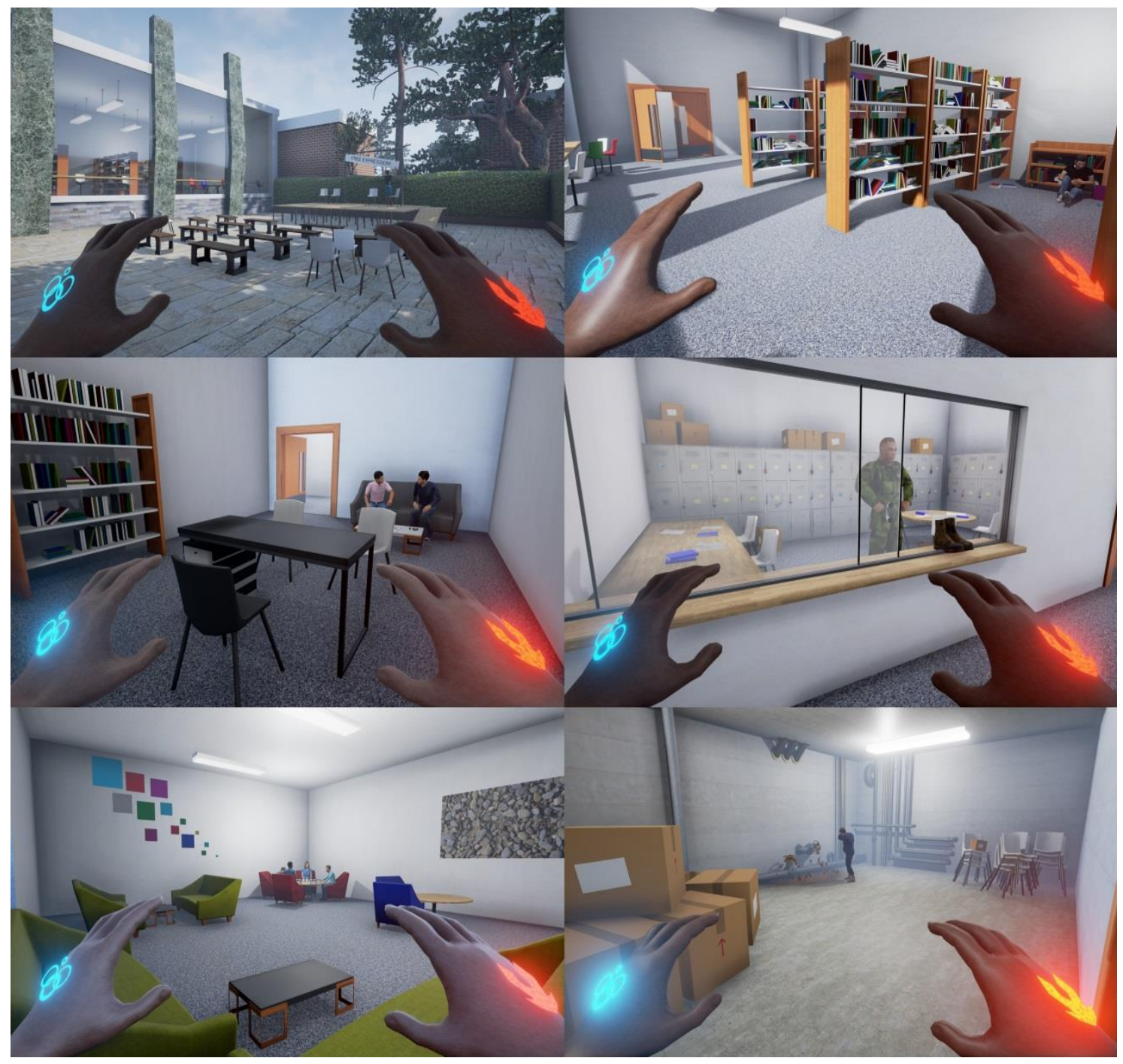

The game mechanics used usability principles such as keeping both the interface and 
gameplay simple and consistent, with enough information presented to players through the game (Sicart, 2008; Pinelle, Wong and Stach, 2008). To assess the usability of the game and engagement within it, once it was complete, game testers were recruited (Schell, 2014). This was to ensure the development of an easy-to-use game that does not require previous experience of video games to play. In addition to this, a small tutorial was created in the game as this was deemed sufficient for the participants to be able to play the game. The aim of this is that the game remains an engaging experience and similar to a typical, commercial first-person RPG. During all stages of the game development, the primary researcher was cautious to avoid including content and factors that may induce moral disengagement (Bandura et al., 1996), such as avoiding dehumanizing the human NPCs that were created for the game. Rewards and motivational techniques were used cautiously, in order to avoid bias in the choices such as avoiding additional information that could be seen as rewarding. This further demonstrates the benefit of creating purpose-made games to measure moral behavior, since in commercial games such as GTA the player is rewarded, encouraged and/or given no other option but to do antisocial actions (i.e. stealing). Therefore, assessing these in-game behaviors may not be valid, whereas purpose-made games can have these biases removed.

Due to binary choices being a typical feature of video games, this same approach was adapted for the format of a decision. The binary choice the participants were presented with, was either to act in an antisocial way (creating a violating situation) or to act in a prosocial way (upholding the MFT foundation and resolving the potentially violating situation). In order for a decision to be made, the player has to interact with the main NPC in the room for the scenario. This would trigger the specific moral foundation related to the scenario. All scenarios involved an object that would either lead to a prosocial or antisocial outcome; this was to avoid hurting NPCs directly and triggering the Harm/Care foundation. For consistency with the other scenarios, even in the Harm/Care scenario, the object (in this case a bookcase) 
is used to harm (see Figure 2). This was important as it meant all the scenarios had an object rather than a character to represent the scenario; thus ensuring all scenarios followed the same format. The violating antisocial choice was placed on the right side of the screen (with controls) and the upholding prosocial choice was placed on the left side of the screen (with controls; see Figure 2). This was selected as commercial games, such as the Mass Effect series, present choices in the same manner. Although counterbalancing the prosocial and antisocial choices between left and right would have reduced the potential response bias, there were two reasons as to why this design was not incorporated. First, it could have been confusing for participants when they made their decisions. Second, programming this into the game would have been considerably time-consuming, adding an additional level of complexity for the development, on a project that already had severe time restrictions. The game was developed to be able to record the choices of each of the six MFT scenarios as well as the time taken to make the choice, as response times for the players' decisions were taken to explore if the decisions and behaviors were more rational or intuitive.

Figure 2. Screenshots of the decision-making process in the purpose-made game for the Care/Harm scenario (Top left: the vignette, top right: the choices presented, bottom left: outcome of the left prosocial choice and bottom right: the outcome of the right antisocial choice). 


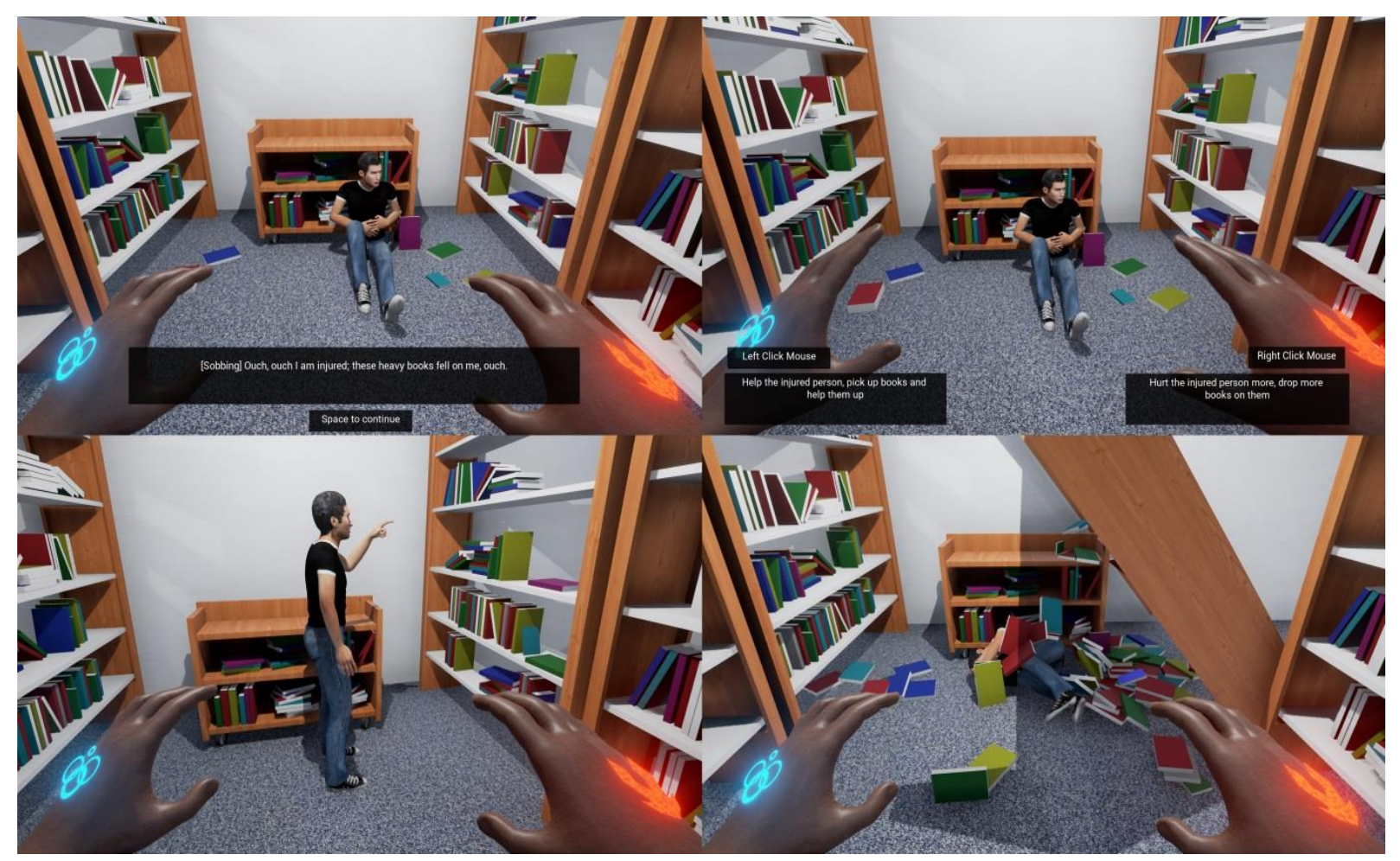

Data collection with the purpose-made game. Once the game had been developed and before the game was used with participants in the main study, participants who could not participate in the main study were gathered to pilot test and review the game. This was carried out for two reasons, the first being that most games need testing for bugs and problems (in this case this included making sure the data was recording properly, a key part of the process) and, second, in order to collect data on how the game was experienced (including usability) compared to commercial games. This also gave the primary researcher experience of the procedure of administering this specific game to participants. The experiment took place in a psychology lab within the University. The participants were observed and recorded playing the game. Before playing the game, participants were given the MFQ (Graham et al., 2008) to measure real life morality and compare this with the decisions made in the video game. After the game was played, participants completed a questionnaire asking them about their experiences and reasons for the decisions made in the game including; the Positive and Negative Affective Schedule (PANAS; Watson \& Clark, 
1999) and Game Engagement Questionnaire (GEQ; Brockmyer et al., 2009) for the current game (rather than general engagement when playing video games). To measure post-game play prosocial and antisocial behavior the Tangram help/hurt task was administered (Saleem, Anderson, \& Barlett, 2015).

Although this is not the focus of this chapter, initial analysis of the results shows that participants demonstrated a preference choosing the prosocial option but these decisions were suggested not to be intuitive. Furthermore, the in-game moral decisions were significantly predicted by level of engagement within the purpose-made game (GEQ; Brockmyer et al., 2009) but not significantly predicted by the MFQ (Graham et al., 2008) and the other postgame measures; PANAS (Watson \& Clark, 1999) and Tangram help/hurt task (Saleem et al., 2015).

\section{Conclusion}

In summary, this chapter discussed the development of a purpose-made game, with the specific intention of using it in research to measure behavioral outcomes through in-game moral decisions. The process of developing a purpose-made game for research required the consideration of psychological theory. Concepts including agency, interactivity and engagement in video games with the implications for moral behavior were discussed. Then, an evaluation of how morality is currently applied in commercial video games and how this game content relates to theories of morality was carried out in order to develop a game. The role and definitions of purpose-made games in research were also explored. Although the focus of this chapter was that of moral behavior and the creation of a game to measure it, purpose-made games can be utilized to measure and investigate many types of psychological phenomena and have significant potential to further the area of psychology and video game research. Specifically for moral choices in video games, other demands of playing video games such as emotional and social involvement, as suggested by Bowman (2016b), could be 
explored. Thus, there are many possible avenues for future research relating to moral behavior when playing video games. 


\section{Ludography}

BioShock series. (2007-2013). Novato, CA: 2K Games.

Call of Duty series (2005-present). Woodland Hills, CA: Activision (Infinity Ward).

Death Race (1976). Fremont, CA: Exidy.

Fable series (2004-2014). Guildford, UK: Lionhead Studios.

Fallout 3. (2008). Rockville, MD: Bethesda.

Grand Theft Auto series (1997-2015). New York City, NY: Rockstar Games.

Half-Life 2. (2004). Bellevue, WA: Valve Software.

Mass Effect series (2007-2012). Edmonton, Canada: BioWare.

NeverWinter Nights (2002). Edmonton, Canada: BioWare.

Operation Flashpoint (2001). Prague, Czechia: Bohemia Interactive.

Spec Ops: The Line (2012). Novato, CA: 2K Games.

Unreal Tournament series (1998-2007). Cary, NC: Epic Games.

Until Dawn (2015). Guildford, UK: Supermassive Games. 


\section{References}

Anderson, C. A., Shibuya, A., Ihori, N., Swing, E. L., Bushman, B. J., Sakamoto, A., Rothstein, H.R., \& Saleem, M. (2010). Violent video game effects on aggression, empathy, and prosocial behavior in eastern and western countries: A meta-analytic review. Psychological Bulletin, 136(2), 151-173. doi 10.1037/a0018251

Anderson, E. F., McLoughlin, L., Watson, J., Holmes, S., Jones, P., Pallett, H., \& Smith, B. (2013). Choosing the Infrastructure for Entertainment and Serious Computer Games a Whiteroom Benchmark for Game Engine Selection. In Proceedings of VS-GAMES 2013: 5th International Conference on Games and Virtual Worlds for Serious Applications (pp. 101-108). IEEE. doi: 10.1109/VS-GAMES.2013.6624223

American Psychological Association . (2015). Task Force on Violent Media Technical Report on the Review of the Violent Video Game Literature. Retrieved from: http://www.apa.org/news/press/releases/2015/08/technical-violent-games.pdf

Bandura, A., Barbaranelli, C., Caprara, G. V., \& Pastorelli, C. (1996). Mechanisms of moral disengagement in the exercise of moral agency. Journal of Personality and Social Psychology, 71(2), 364. doi: 10.1037/0022-3514.71.2.364

Bowman, N. D. (2016a). The rise (and refinement) of moral panic. In R. T. Kowert (Ed.), The Video Game Debate: Unravelling the Physical, Social, and Psychological Effects of Video Games (pp. 22-38). New York: Routledge.

Bowman, N. D. (2016b). Video gaming as co-production. In R. Lind (Ed.), Produsing 2.0: The intersection of audiences and production in a digital world (Vol. 2, pp. 107-123). New York: Peter Lang Publishing.

Bowman, N. D., Weber, R., Tamborini, R., \& Sherry, J. L. (2013). Facilitating game play: How others affect performance at and enjoyment of video games. Media Psychology, 16(1), 39-64. doi: 10.1080/15213269.2012.7 
Brockmyer, J. H., Fox, C. M., Curtiss, K. A., McBroom, E., Burkhart, K. M., \& Pidruzny, J. N. (2009). The development of the Game Engagement Questionnaire: A measure of engagement in video game-playing. Journal of Experimental Social Psychology, 45(4), 624-634. doi: 10.1016/j.jesp.2009.02.016

Christiansen, P. (2017). Designing ethical systems for videogames. In Proceedings of the 12th International Conference on the Foundations of Digital Games (p. 21). ACM. doi: $10.1145 / 3102071.3102088$

Clifford, S., Iyengar, V., Cabeza, R., \& Sinnott-Armstrong, W. (2015). Moral foundations vignettes: a standardized stimulus database of scenarios based on moral foundations theory. Behavior Research Methods, 47(4), 1178-1198. doi: 10.3758/s13428-014$0551-2$

Connolly, T. M., Boyle, E. A., MacArthur, E., Hainey, T., \& Boyle, J. M. (2012). A systematic literature review of empirical evidence on computer games and serious games. Computers \& Education, 59(2), 661-686. doi: 10.1016/j.compedu.2012.03.004

Consalvo, M. (2009). There is No Magic Circle. Games and Culture, 4(4), 408-417. doi: $10.1177 / 1555412009343575$

De Lope, R. P., \& Medina-Medina, N. (2016). A comprehensive taxonomy for serious games. Journal of Educational Computing Research, 55(5), 629-672. doi: $10.1177 / 0735633116681301$

Dill K. E., Gentile D. A., Richter W. A., Dill J. C. (2005). Violence, sex, race and age in popular video games: A content analysis. In Cole E., Henderson Daniel J. (Eds.), Featuring females: Feminist analyses of the media, pp. 115-130. Washington, DC: American Psychological Association.

Elson, M., \& Quandt, T. (2016). Digital games in laboratory experiments: Controlling a complex stimulus through modding. Psychology of Popular Media Culture, 5(1), 52. 
doi: $10.1037 / \mathrm{ppm} 0000033$

Ferguson, C. J. (2007). Evidence for publication bias in video game violence effects

literature: A meta-analytic review. Aggression and Violent Behavior, 12(4), 470-482. doi: 10.1016/j.avb.2007.01.001

Frasca, G. (2001). Rethinking agency and immersion: video games as a means of consciousness-raising. Digital Creativity, 12(3), 167-174. doi: 10.1076/digc.12.3.167.3225

Graham, J., Haidt, J., \& Nosek, B. (2008). The Moral Foundations Questionnaire (MFQ). Retrieved from: http://www.moralfoundations.org/questionnaires

Greig, S., Muzyka, R., Ohlen, J., Oster, T., \& Zeschuk, G. (2002). Postmortem: Bioware's Neverwinter Nights. Retrieved from: http://web.archive.org/web/20121111000517/http://www.gamasutra.com/view/feature /131327/postmortem_biowares_neverwinter_.php?page $=4$

Grizzard, M., Tamborini, R., Lewis, R. J., Wang, L., \& Prabhu, S. (2014). Being Bad in a Video Game Can Make Us More Morally Sensitive. CyberPsychology, Behavior \& Social Networking, 17(8), 499-504. doi: 10.1089/cyber.2013.0658

Grizzard, M., Tamborini, R., Sherry, J. L., \& Weber, R. (2016). Repeated Play Reduces Video Games’ Ability to Elicit Guilt: Evidence from a Longitudinal Experiment. Media Psychology, 20(2) 267-290. doi:10.1080/15213269.2016.1142382

Grodal, T. (2000). Video games and the pleasure of control. In D. Zillman \& P. Vorderer (Eds.), Media entertainment: The psychology of its appeal, (pp. 197-213). Mahwah, NJ: Lawrence Erlbaum.

Guinness World Records. (2014). Most successful video game engine. Retrieved from: http://www.guinnessworldrecords.com/world-records/most-successful-game-engine Haidt, J. (2001). The emotional dog and its rational tail: a social intuitionist approach to 
moral judgment. Psychological Review, 108(4), 814. doi: 10.1037/0033-

295X.108.4.814

Haidt, J. (2012). The righteous mind: Why good people are divided by politics and religion:

New York: Pantheon Books.

Haidt, J., \& Joseph, C. (2004). Intuitive ethics: How innately prepared intuitions generate culturally variable virtues. Daedalus, 133(4), 55-66. doi: 10.1162/0011526042365555

Haidt, J., \& Joseph, C. (2007). The moral mind: How five sets of innate intuitions guide the development of many culture-specific virtues, and perhaps even modules. The Innate Mind, 3, 367-391. doi: 10.1093/acprof:oso/9780195332834.003.0019

Hartmann, T. (2011). Players' experiential and rational processing of virtual violence. In K. Poels \& S. Malliet (Eds.), Moral issues in digital game play (pp. 135-150). Leuven, Belgium: Acco.

Hartmann, T., \& Vorderer, P. (2010). It's okay to shoot a character: Moral disengagement in violent video games. Journal of Communication, 60(1), 94-119. doi: 10.1111/j.14602466.2009.01459.x

Heron, M. J., \& Belford, P. H. (2014). Do You Feel Like a Hero Yet? Externalised Morality in Video Games. Journal of Games Criticism, 1(2), 1-22.

Huizinga, J. (1949). Homo Ludens: A Study of the Play Element in Culture: Boston: Beacon Press.

Jerabeck, J. M., \& Ferguson, C. J. (2013). The influence of solitary and cooperative violent video game play on aggressive and prosocial behavior. Computers in Human Behavior, 29(6), 2573-2578. doi: 10.1016/j.chb.2013.06.034

Joeckel, S., Bowman, N. D., \& Dogruel, L. (2012). Gut or Game? The Influence of Moral Intuitions on Decisions in Video Games. Media Psychology, 15(4), 460-485. doi: $10.1080 / 15213269.2012 .727218$ 
Joeckel, S., Bowman, N. D., \& Dogruel, L. (2013). The Influence of Adolescents' Moral Salience on Actions and Entertainment Experience in Interactive Media. Journal of Children and Media, 7(4), 480-506. doi: 10.1080/17482798.2013.781513

Kocurek, C. (2012). The agony and the Exidy: a history of video game violence and the legacy of Death Race. Game Studies, 12(1). Retrieved from: http://gamestudies.org/1201/articles/carly_kocurek

Lange, A. (2014). 'You're Just Gonna Be Nice': How Players Engage with Moral Choice Systems. Journal of Games Criticism, 1(1), 1-16.

Lewis, M., \& Jacobson, J. (2002). Game engines in scientific research: Introduction to special issue. Communications of the ACM, 45(1), 27-31.

Limperos, A., Downs, E., Ivory, J., \& Bowman, N. D. (2013). Leveling up: A review of emerging trends and suggestions for the next generation of communication research investigating video games' effects. Annals of the International Communication Association, 37(1), 349-377. doi: 10.1080/23808985.2013.11679155

McKernan, B. (2013). The Morality of Play Video Game Coverage in The New York Times From 1980 to 2010. Games and Culture, 8(5), 307-329. doi: $10.1177 / 1555412013493133$

Miller, P. (2013). Jesse Schell's search for the Shakespeare of video games. Gamasutra. Retrieved from: http://www.gamasutra.com/view/news/189370/Jesse_Schells_search_for_the_Shakes peare_of_video_games.php

Oliver, M. B., Bowman, N. D., Woolley, J. K., Rogers, R., Sherrick, B. I., \& Chung, M. Y. (2015). Video games as meaningful entertainment experiences. Psychology of Popular Media Culture, 5. doi: 10.1037/ppm0000066

Pinelle, D., Wong, N., \& Stach, T. (2008, April). Heuristic evaluation for games: usability 
principles for video game design. In Proceedings of the SIGCHI Conference on Human Factors in Computing Systems (pp. 1453-1462). ACM. doi:

\section{$10.1145 / 1357054.1357282$}

Prot, S., Gentile, D. A., Anderson, C. A., Suzuki, K., Swing, E., Lim, K. M., Horiuchi, Y., Jelic, M., Krahé, B., Liuqing, W., Liau, A. K., Khoo, A., Petrescu, P. D., Sakamoto,A., Tajima, S., Toma, R.A., Warburton, W., Zhang, X., \& Lam B. C. P. (2014). Long-term relations among prosocial-media use, empathy, and prosocial behavior. Psychological Science, 25(2), 358-368. doi: 10.1177/0956797613503854

Salen, K., \& Zimmerman, E. (2004). Rules of play: Game design fundamentals. Cambridge: MIT press.

Saleem, M., Anderson, C. A., \& Barlett, C. P. (2015). Assessing helping and hurting behaviors through the Tangram help/hurt task. Personality and Social Psychology Bulletin, 41(10), 1345-1362. doi: 10.1177/0146167215594348

Schell, J. (2014). The Art of Game Design: A book of lenses: CRC Press.

Sherry, J. L. (2001). The effects of violent video games on aggression. Human communication research, 27(3), 409-431. doi: 10.1111/j.1468-2958.2001.tb00787.x

Sherry, J. L. (2004). Flow and media enjoyment. Communication Theory, 14(4), 328-347. Doi: 10.1111/j.1468-2885.2004.tb00318.x

Sicart, M. (2008). Defining game mechanics. Game Studies, 8(2), 1-14.

Steuer, J. (1992). Defining virtual reality: Dimensions determining telepresence. Journal of Communication, 42(4), 73-93. doi: 10.1111/j.1460-2466.1992.tb00812.x

Stromer-Galley, J. (2004). Interactivity-as-product and interactivity-as-process. The Information Society, 20, 391-394. doi:10.1080/01972240490508081.

Tamborini, R. (2011). Moral intuition and media entertainment. Journal of Media Psychology, (23), 39-45. doi: 10.1027/1864-1105/a000031 
Tamborini, R., \& Bowman, N. D. (2010). Presence in video games. Immersed in media: Telepresence in everyday life, 87-109. New York: Routledge.

Tamborini, R. C. (Ed.). (2013). Media and the moral mind. London: Routledge.

Tamborini, R., Bowman, N. D., Prabhu, S., Hahn, L., Klebig, B., Grall, C., \& Novotny, E. (2016). The effect of moral intuitions on decisions in video game play: The impact of chronic and temporary intuition accessibility. New Media \& Society, doi: $10.1177 / 1461444816664356$.

Thomas, N. (2006). Video Games as Moral Universes. TOPIA: Canadian Journal of Cultural Studies, (11), 101-115.

Watson, D., \& Clark, L. A. (1999). The PANAS-X: Manual for the Positive and Negative Affect Schedule - Expanded form. Iowa City: University of Iowa.

Weaver, A. J., \& Lewis, N. (2012). Mirrored Morality: An Exploration of Moral Choice in Video Games. CyberPsychology, Behavior \& Social Networking, 15(11), 610-614. doi: 10.1089/cyber.2012.0235

Young, G. (2013). Enacting taboos as a means to an end; but what end? On the morality of motivations for child murder and paedophilia within gamespace. Ethics and Information Technology, 15(1), 13-23. doi: 10.1007/s10676-012-9306-x 